\title{
Clinical implications of Delphian lymph node metastasis in papillary thyroid carcinoma
}

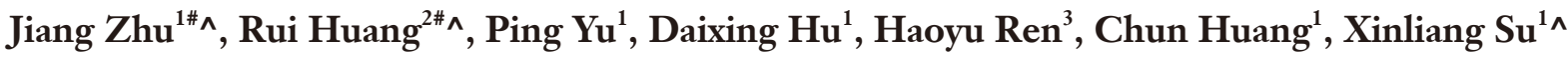 \\ ${ }^{1}$ Department of Endocrine and Breast Surgery, The First Affiliated Hospital of Chongqing Medical University, Chongqing, China; ${ }^{2}$ Department \\ of Anesthesiology, The First Affiliated Hospital of Chongqing Medical University, Chongqing, China; ${ }^{3}$ Department of General, Visceral, and \\ Transplant Surgery, Ludwig-Maximilians-University, Munich, Germany \\ Contributions: (I) Conception and design: J Zhu, X Su; (II) Administrative support: X Su; (III) Provision of study materials or patients: J Zhu, X Su; (IV) \\ Collection and assembly of data: J Zhu, R Huang, P Yu, C Huang, D Hu; (V) Data analysis and interpretation: J Zhu, R Huang, X Su, H Ren; (VI) \\ Manuscript writing: All authors; (VII) Final approval of manuscript: All authors. \\ \#These authors contributed equally to this work. \\ Correspondence to: Xinliang Su. Department of Endocrine and Breast Surgery, The First Affiliated Hospital of Chongqing Medical University, 1 Youyi \\ Rd, Chongqing 400016, China. Email: suxinliang@21cn.com.
}

Background: As is known, identifying risk factors precisely for lymph node metastasis (LNM) plays a vital role in initial treatment for papillary thyroid carcinoma (PTC). Nonetheless, whether Delphian lymph node (DLN) metastasis has value in predicting LNM remains an open question. This study covered a sample of 1,575 patients, which is the largest sample group so far, aiming to assess the predictive validity of DLN metastasis in PTC.

Methods: This retrospective cohort study was conducted with 1,575 eligible PTC patients who underwent thyroid operation between July 2013 and December 2018 and clinicopathologic parameters of patients with DLN metastasis were compared with those without DLN metastasis.

Results: The incidence of DLN metastasis, according to our research samples, is $24.4 \%$ (384/1,575 patients). And results show that DLN positivity was closely associated with adverse prognostic factors including younger age, larger tumor size, extrathyroid extension, tumor location in the isthmus or upper lobe of the thyroid, number of LNM >5, higher recurrence. After carefully adjusting important confounding factors, we find that in multivariate logistic regression analyses, DLN metastasis is an independent predictor for both central LNM (CLNM, adjusted $\mathrm{OR}=7.81, \mathrm{P}<0.001$ ) and lateral LNM (LLNM, adjusted OR =3.40, $\mathrm{P}<0.001)$. Moreover, the stratified analyses also show convincing evidence of a positive correlation between DLN metastasis and LNM in levels II-IV in the vast majority of subgroups.

Conclusions: The present study suggests that DLN metastasis is an independent risk factor for CLNM and LLNM of levels II-IV. The cervical lymph nodes should be meticulously evaluated to guide tailored treatment during operation in patients with DLN involvement.

Keywords: Papillary thyroid carcinoma (PTC); Delphian lymph nodes metastasis; central lymph node metastasis (CLNM); lateral lymph node metastasis (LLNM)

Submitted May 22, 2020. Accepted for publication Sep 18, 2020.

doi: $10.21037 / g s-20-521$

View this article at: http://dx.doi.org/10.21037/gs-20-521

^ ORCID: Jiang Zhu: 0000-0003-4194-2355; Rui Huang: 0000-0002-3342-135X; Xinliang Su: 0000-0001-5792-1407. 


\section{Introduction}

The incidence of papillary thyroid carcinoma (PTC) has increased sharply worldwide in recent decades (1). Generally, PTC has an excellent prognosis, but neck lymph node metastases (LNM) are quite common, which increases the risk of tumor relapse and reduce long-term survival (2-4). Evaluating LNM accurately is thus essential for appropriate initial surgical management $(5,6)$. The Delphian lymph node (DLN) (prelaryngeal lymph node) was named by the "Oracle of Delphi" (7), the most famous religious prophet in the temple of Apollo of ancient Greek times. Several studies have reported that DLN metastasis might be predictive of widespread nodal involvement (7-9). A recent meta-analysis reported that DLN positivity is valuable for predicting central and lateral LNM (10). However, the population included, study methods and results reported were heterogeneous, thus the clinical significance of DLN metastasis has yet to be independently evaluated. Therefore, to clarify the clinical implications of DLN positivity, the present study investigated the clinicopathologic characteristics of DLN metastasis in patients with PTC.

We present the following article in accordance with the STROBE reporting checklist (available at http://dx.doi. org/10.21037/gs-20-521).

\section{Methods}

\section{Patients selection}

This retrospective study was conducted with clinical information collected at the Department of Endocrine and Breast Surgery, The First Affiliated Hospital of Chongqing Medical University from July 2013 to December 2018. This study was conducted in accordance with the Declaration of Helsinki (as revised in 2013), and approved by ethical review board of The First Affiliated Hospital of Chongqing Medical University (reference number: 2020182). Informed consent was obtained from all participants. Clinical information, such as tumor diameter, clinical $\mathrm{N}$ stage, gender, age, LNM and other relevant data of patients were derived from the electronic medical records. Inclusion criteria were patients with histologically confirmed PTC who underwent thyroid surgery and had complete medical records and follow-up information while patients who had neck surgery before and those with incomplete medical records and distant metastasis were exclusions.

\section{Surgery methods}

In our center, we routinely perform unilateral lobectomy with ipsilateral central compartment dissection. Total thyroidectomy is performed when tumor size $>20 \mathrm{~mm}$, ETE, bilaterality or abnormal lymphadenopathy by intraoperative frozen section examination. Selective lateral compartment dissection of levels II-IV is performed in patients with suspicious lymph nodes involvement on preoperative images. Meanwhile, when intraoperative frozen biopsy showed $\geq 3$ central lymph node (CLN) metastases including positive DLN, ETE, tumor located in the upper lobe, those risk factor above appear simultaneously, we also performed lateral lymph node dissection.

All surgeries were performed according to a standard procedure (11): we inject nanocarbon suspension into the thyroid gland to distinguish the stained lymph nodes from fatty tissues facilitating nodal dissection. CLN were divided into 3 nodal sites: DLN, pretracheal LN and paratracheal LN. First, soft tissue in the prelaryngeal region was deliberately excised and labeled as the DLN, then pretracheal LN was removed and labeled as pretracheal LN. Those two groups were immediately and separately sent for intraoperative frozen section examination while we performed lobectomy and ipsilateral paratracheal LN dissection. The paratracheal LN were also sent for frozen section examination as mentioned above. Surgical specimens were all sent for postoperative histopathologic diagnosis by three pathologists.

\section{Follow-up}

Patients were followed up 12-78 months. The locoregional recurrence was defined as any newly detected tumor or metastatic LN on fine-needle aspiration (FNA) or on the pathologic diagnosis after reoperation.

\section{Statistical analysis}

Continuous variables are presented as the means \pm standard deviations (SDs) or the medians and interquartile range (IQR) and categorical variables as numbers (percentage). The baseline characteristics between subjects with and without DLN metastasis were compared using independentsamples $t$-test or Mann-Whitney $\mathrm{U}$ test for continuous variables, the chi-squared test and Fisher's exact test for categorical variables. Four multivariate logistic regression 


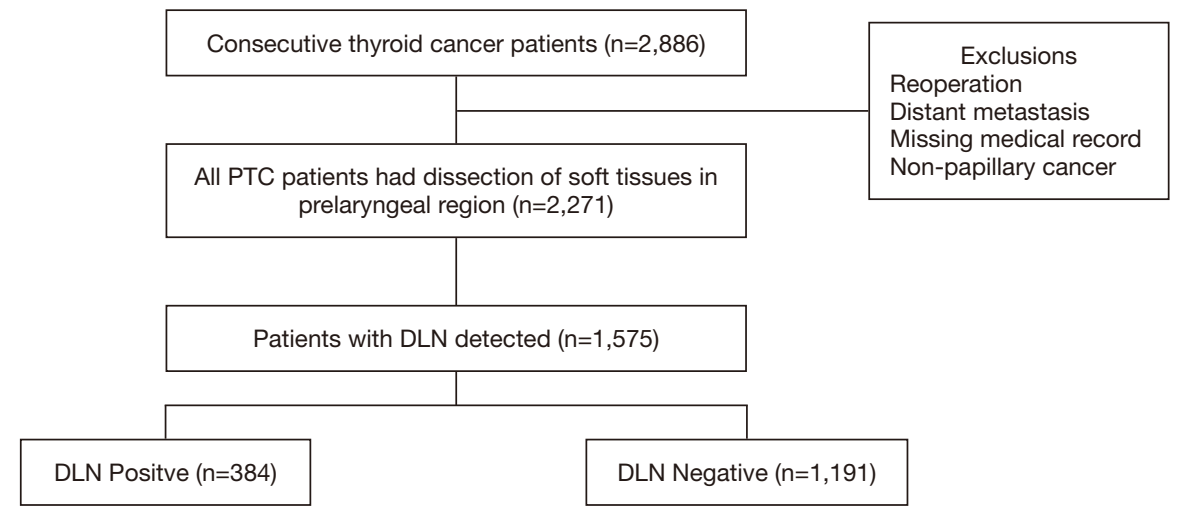

Figure 1 Flow diagram of patients selection. DLN, Delphian lymph node; PTC, papillary thyroid carcinoma.

models were constructed in total to assess the association of DLN metastasis with central lymph node metastasis (CLNM) and lateral lymph node metastasis (LLNM) including Model 1, unadjusted; Model 2, adjusted for sex, age; Model 3, adjusted for sex, age, tumor size, location, multifocality, ETE and clinical nodal status; and Model 4 adjusted for sex, age, tumor size, location, multifocality, ETE and CLNM. We conducted stratified analyses to explore the relationship between DLN metastasis and level II-IV metastasis by age, sex, tumor size and location, CLN status, ETE. Statistical analyses were all conducted with the $\mathrm{R}$ software version 3.6.2 (www.r-project.org). The ggplot2 and forest plot packages were used to visualize data. $\mathrm{P}<0.05$ was considered significant.

\section{Results}

\section{Clinicopathologic characteristics of 1,575 PTC patients}

In a total of 2,886 consecutive patients who underwent thyroid surgery during the study period, 2,271 patients were included as eligible data for this retrospective analysis, among which DLN was detected and routinely removed in 1,575 patients $(1,575 / 2,271,69.4 \%)$ (Figure 1). The characteristics of patients and the distribution of the groups with and without DLN metastasis are shown in Table 1. Among the 1,575 patients [429 male and 1,146 female; median age (IQR) 41 years (32-49 years)], 384 (24.4\%) patients were confirmed positive for DLN metastasis while preoperative ultrasonography showed 232 (14.7\%) patients were $\mathrm{cN} 1$. Central and lateral LNM were confirmed in $56.8 \%$ and $31.8 \%$ of patients after surgery, respectively. The mean follow-up period was 34 months, recurrence occurred in $2.0 \%(31 / 1,575)$ of cases.
The DLN-positive group had significantly higher percentages of male patients, age $<55$ years, extrathyroid extension as compared with the DLN-negative group (all $\mathrm{P}<0.001)$. The proportions of papillary thyroid microcarcinoma significantly differed between patients with DLN metastasis and patients without DLN metastasis (38.3\% vs. $68.9 \%, \mathrm{P}<0.001)$. DLN metastasis was associated with CLNM (90.6\% vs. $45.8 \%, \mathrm{P}<0.001)$, LLNM (68.0\% vs. 20.2\%, $\mathrm{P}<0.001)$. Patients with positive DLN involvement were more likely to have tumor located in upper portion/isthmus $(36.5 \%$ vs. $25.1 \%, \mathrm{P}<0.001)$ and $>5$ metastatic lymph nodes $(54.7 \%$ vs. $10.7 \%, \mathrm{P}<0.001)$. Level II (36.7\% vs. 6.9\%, $\mathrm{P}<0.001)$, Level III $(53.4 \%$ vs. $13.5 \%, \mathrm{P}<0.001)$ and Level IV metastasis $(42.2 \%$ vs. $11.0 \%, \mathrm{P}<0.001)$ were more common in the DLN-positive group. There was no significant difference in Hashimoto thyroiditis between the two groups. Compared with the DLN-negative group, the DLN-positive group showed a higher rate of recurrence ( $3.6 \%$ vs. $1.4 \%, \mathrm{P}=0.010)$.

As shown in Figure 2, the median tumor size and number of metastatic lymph nodes in the DLN-positive group were both significantly higher than that in the DLN-negative group (all $\mathrm{P}<0.05$ ), regardless of male or female. The incidence of CLNM according to the clinical $\mathrm{N}$ stage is presented in Figure $3 \mathrm{~A}$. cN1: central lymph node metastatic rate in groups with DLN metastasis and without DLN metastasis were $98.1 \%, 68.8 \%$, respectively $(\mathrm{P}<0.001)$. cN0: central lymph node metastatic rate were $87.7 \%, 43.2 \%$, respectively $(\mathrm{P}<0.001)$. We found that for patients with stageT1-T2, noninvasive, cN0 tumors, the DLN-positive group yielded significantly higher incidence of CLNM as compared with DLN-negative group (86.4\% vs. 42.7\%, $\mathrm{P}<0.001$ ) (Figure 3B). 
Table 1 Baseline characteristics of included patients with papillary thyroid carcinomas

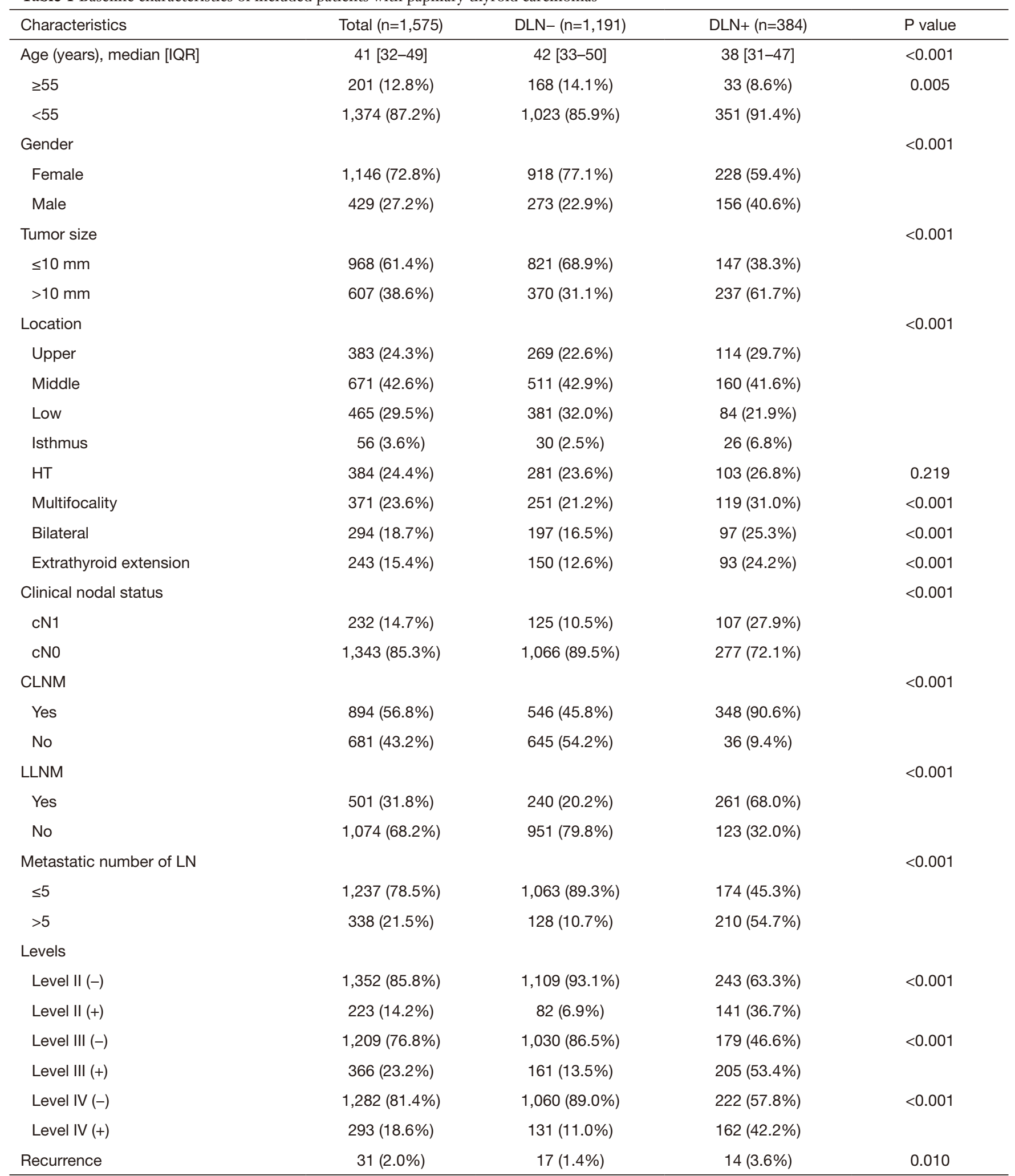

LN, lymph node; DLN, Delphian lymph node; IQR, interquartile range; HT, Hashimoto's thyroiditis; CLNM, central lymph node metastasis; LLNM, lateral lymph node metastasis. 

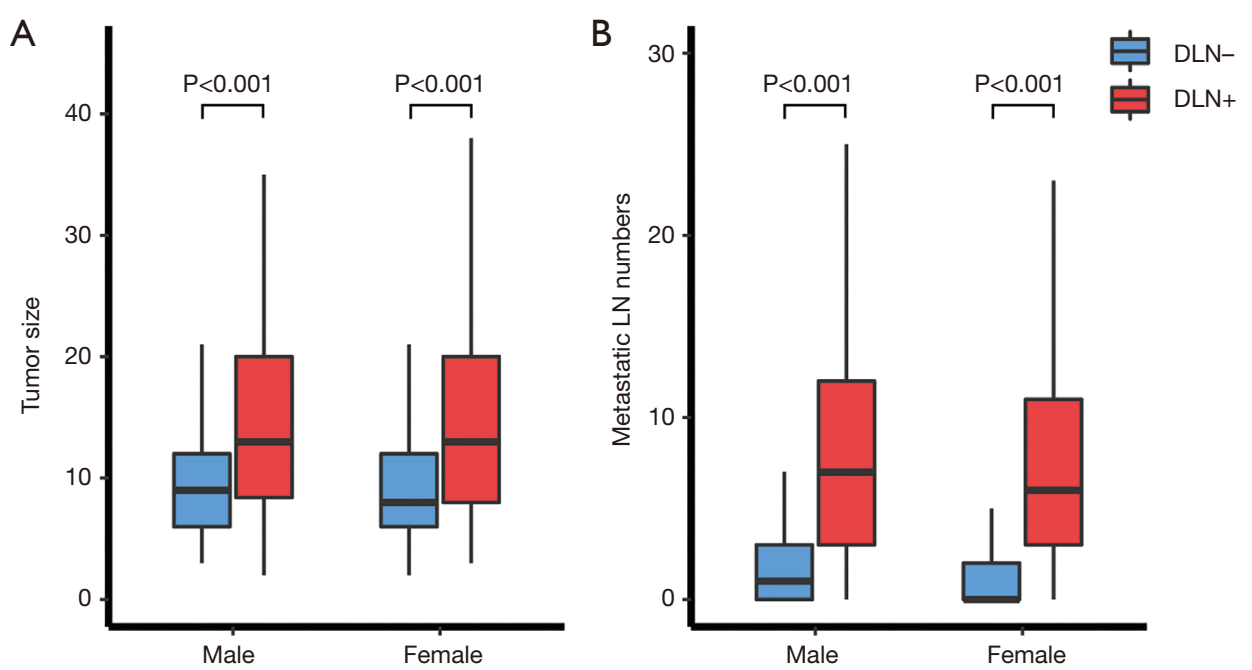

Figure 2 Comparison of tumor size (A) and the number of metastatic LN (B) between the DLN-negative group and the DLN-positive group. LN, lymph node; DLN, Delphian lymph node.

A

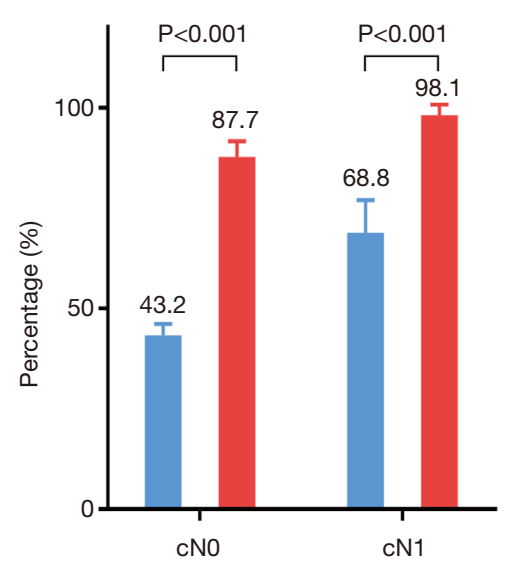

B T1-T2, noninvasive, cNO cases

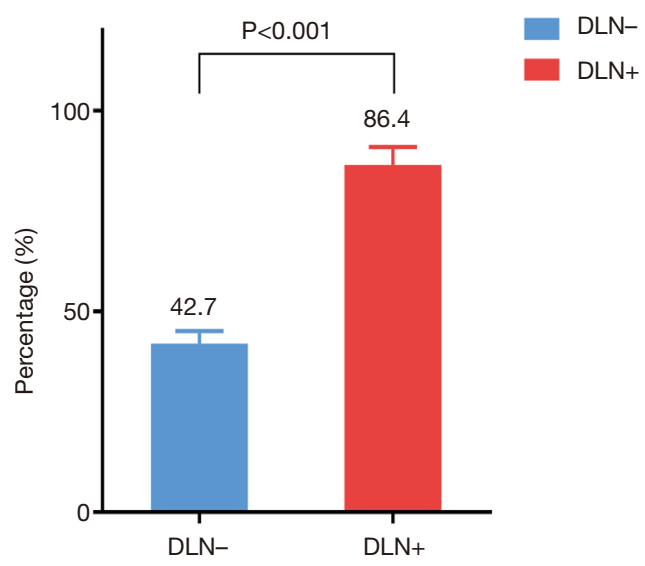

Figure 3 Incidence of central lymph node metastasis in all patients (A) and in patients with T1-T2, noninvasive, cN0 tumors (B) according to clinical nodal and the DLN status. cN0, clinical lymph node negative; cN1, clinical central or lateral lymph node positive; DLN, Delphian lymph node.

Table 2 displays the association between DLN status and CLNM, LLNM. Univariate analysis indicated that DLN metastasis was positively associated with CLNM. After adjustment for tumor location, size, multifocality, age, sex, extrathyroid extension and clinical nodal status, associations were still maintained (adjusted $\mathrm{OR}=7.81$; 95\% CI, 5.34-11.43). Likewise, DLN metastasis was a statistically independent risk factor for LLNM (adjusted $\mathrm{OR}=3.51$; 95\% CI, 2.56-4.79). Furthermore, the stratified analyses revealed that DLN metastasis was statistically and independently correlated with levels II, III, IV metastasis in the vast majority of subgroups (Figures 4,5,6). Interestingly, none of the isthmus tumors in DLN-negative group had level II or level III metastasis.

\section{Discussion}

This study analyzed real-world data of 1,575 PTC patients. 
Table 2 Multivariate logistic models for CLNM and LLNM

\begin{tabular}{|c|c|c|c|c|c|}
\hline & DLN status & \multicolumn{4}{|c|}{ OR $(95 \% \mathrm{Cl})$} \\
\hline \multirow[t]{2}{*}{ CLNM } & Negative & 1 (reference) & 1 (reference) & 1 (reference) & \\
\hline & Positive & $11.42(7.96-16.39)$ & $10.36(7.20-14.92)$ & $7.81(5.34-11.41)$ & \\
\hline LLNM & Negative & 1 (reference) & 1 (reference) & & 1 (reference) \\
\hline
\end{tabular}

${ }^{*}$, unadjusted. ${ }^{\dagger}$, adjusted for age, sex. ${ }^{\ddagger}$, adjusted for age, sex, tumor size, location, multifocality, extrathyroid extension and clinical nodal status. ${ }^{\S}$, adjusted for age, sex, tumor size, location, multifocality, extrathyroid extension and central lymph node metastasis. DLN, Delphian lymph node; CLNM, central lymph node metastasis; LLNM, lateral lymph node metastasis; OR, odds ratio; Cl, confidence interval.

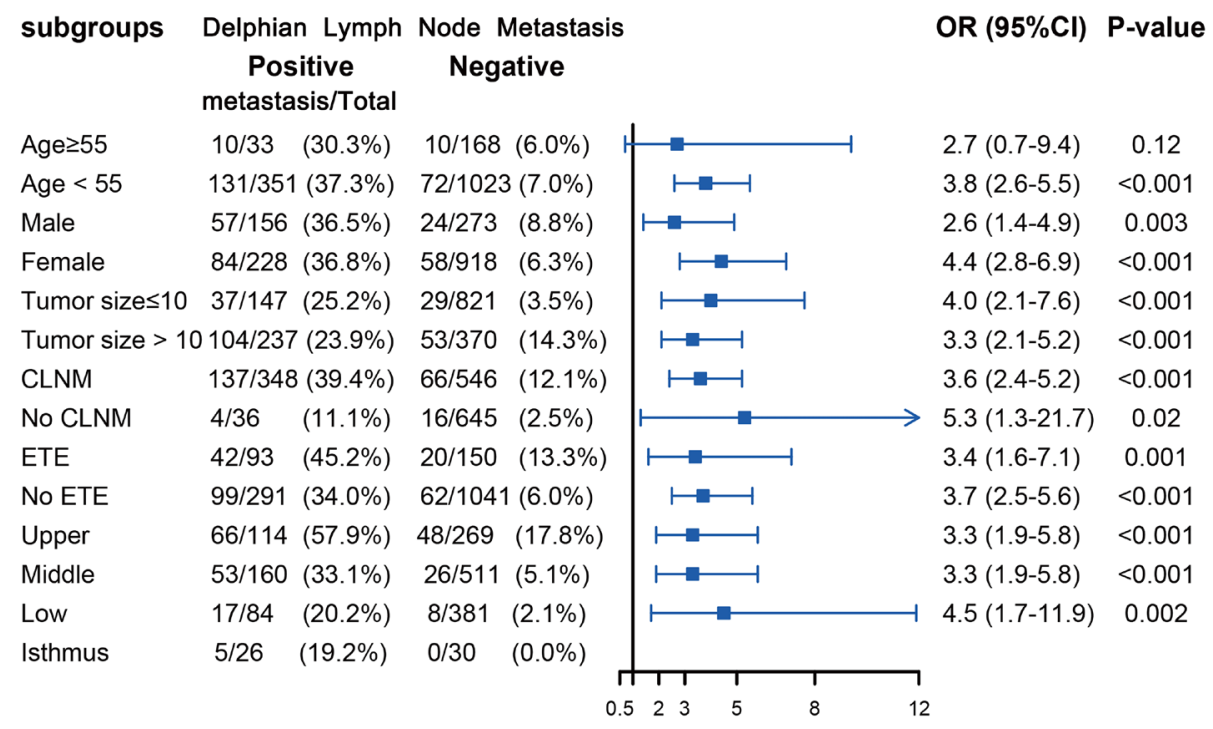

Figure 4 Subgroup analysis of association between level II metastasis and DLN metastasis according to baseline characteristics. DLN, Delphian lymph node; ETE, extrathyroid extension; CLNM, central lymph node metastasis; OR, odds ratio; CI, confidence interval.

The results revealed that DLN metastasis was significantly associated with poor clinicopathologic characteristics of PTC, including larger tumor size, ETE, multifocality, higher rerecurrence. Furthermore, DLN involvement was highly predictive of further central compartment disease, irrespective of $\mathrm{cN} 0$ or $\mathrm{cN} 1$. It was also an independent predictor of lateral compartment disease containing levels II, III, IV, and this positive correlation was evident across most subgroups after meticulous adjustments.

LNM is an important factor of recurrent disease, which places a huge burden on both surgeons and patients (12). Many clinicopathological factors associated with LNM including tumor location, DLN status have not been carefully considered in risk stratification in any recommendations or guidelines to help intraoperative decision-making for tailored surgery. Previous several similar studies indicated that DLN involvement was associated with adverse prognostic factors $(8,9)$. However, their sample size is so small (with no more than 500 cases) and most are univariate analyses, which may not generate robust results. Recent meta-analysis had similar conclusions, albeit limited by the heterogeneity of the studies and data in the literature regarding the clinical significance of DLN metastasis were insufficient. This study is expected to contribute significant data to this topic.

Scholars in North America and European countries 


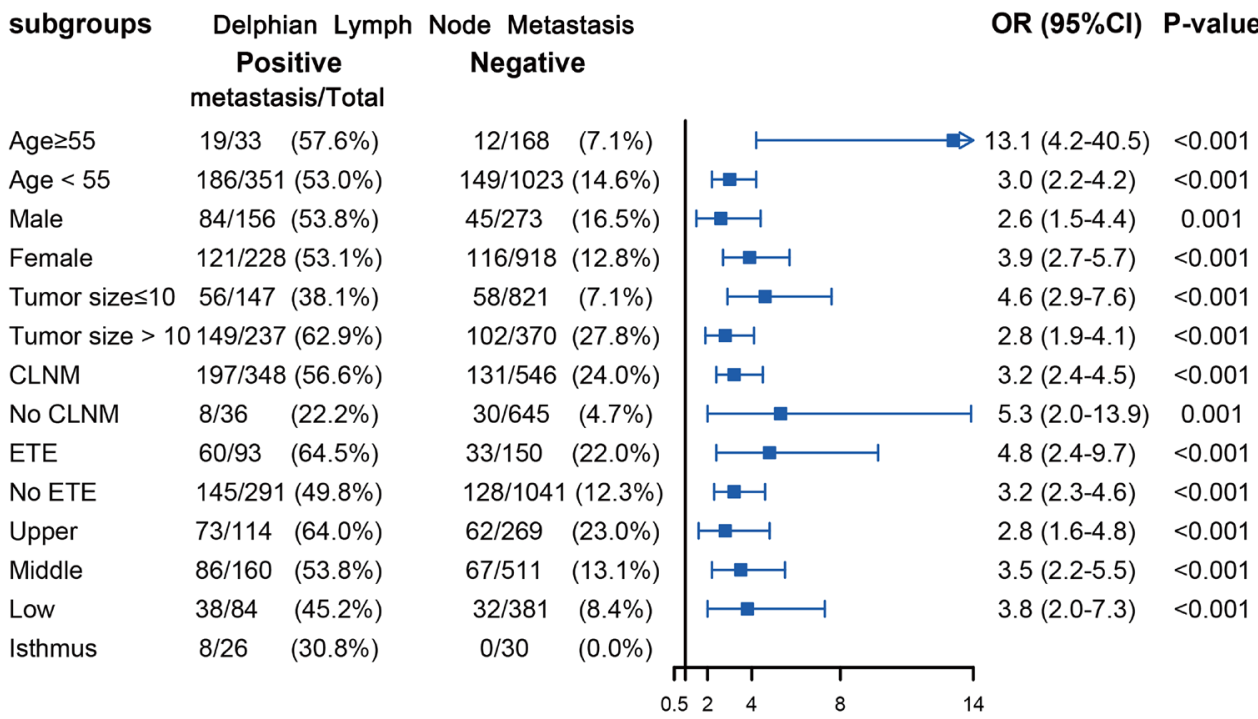

Figure 5 Subgroup analysis of association between level III metastasis and DLN metastasis according to baseline characteristics. DLN, Delphian lymph node; ETE, extrathyroid extension; CLNM, central lymph node metastasis; OR, odds ratio; CI, confidence interval.

\begin{tabular}{|c|c|c|c|c|c|c|}
\hline subgroups & $\begin{array}{r}\text { Delphian } \\
\text { Pos } \\
\text { metastas }\end{array}$ & $\begin{array}{l}\text { Lymph } \\
\text { itive } \\
\text { sis/Total }\end{array}$ & $\begin{array}{c}\text { Node Metastasis } \\
\text { Negative }\end{array}$ & & OR (95\%Cl) & P-value \\
\hline Age $\geq 55$ & $15 / 33$ & $(45.5 \%)$ & $12 / 168 \quad(7.1 \%)$ & $\longmapsto$ & $7.1(2.5-20.5)$ & $<0.001$ \\
\hline Age $<55$ & $147 / 351$ & $(41.9 \%)$ & $119 / 1023(11.6 \%)$ & $\mapsto-1$ & $2.3(1.7-3.2)$ & $<0.001$ \\
\hline Male & $69 / 156$ & $(44.2 \%)$ & $30 / 273 \quad(11.0 \%)$ & $\longmapsto$ & $2.7(1.5-4.8)$ & $<0.001$ \\
\hline Female & $93 / 228$ & $(40.8 \%)$ & $101 / 918 \quad(11.0 \%)$ & $\mapsto-1$ & $2.5(1.7-3.6)$ & $<0.001$ \\
\hline Tumor size $\leq 10$ & $44 / 147$ & $(29.9 \%)$ & $50 / 821 \quad(6.1 \%)$ & $\longmapsto$ & $3.2(1.9-5.4)$ & $<0.001$ \\
\hline Tumor size $>10$ & $0118 / 237$ & $(49.8 \%)$ & $81 / 370 \quad(21.9 \%)$ & $\mapsto-1$ & $2.2(1.5-3.2)$ & $<0.001$ \\
\hline CLNM & $157 / 348$ & $3(45.1 \%)$ & $116 / 546 \quad(21.2 \%)$ & $\mapsto-1$ & $2.4(1.7-3.3)$ & $<0.001$ \\
\hline No CLNM & $5 / 36$ & $(13.9 \%)$ & $15 / 645 \quad(2.3 \%)$ & $\longmapsto$ & $6.0(1.9-18.7)$ & 0.002 \\
\hline ETE & $48 / 93$ & $(51.6 \%)$ & $33 / 150 \quad(22.0 \%)$ & $\longmapsto$ & $2.2(1.1-4.2)$ & 0.023 \\
\hline No ETE & $114 / 291$ & $(39.2 \%)$ & $98 / 1041(9.4 \%)$ & $\mapsto-1$ & $2.7(1.9-3.8)$ & $<0.001$ \\
\hline Upper & $56 / 114$ & $(49.1 \%)$ & $40 / 269 \quad(14.9 \%)$ & $\longmapsto$ & $2.4(1.4-4.3)$ & 0.002 \\
\hline Middle & $62 / 160$ & $(38.8 \%)$ & $55 / 511 \quad(10.8 \%)$ & $\longmapsto-1$ & $2.2(1.3-3.6)$ & 0.002 \\
\hline Low & $35 / 84$ & $(41.7 \%)$ & $35 / 381 \quad(9.2 \%)$ & $\longmapsto$ & $2.9(1.6-5.5)$ & 0.002 \\
\hline Isthmus & $9 / 26$ & $(34.6 \%)$ & $1 / 30 \quad(3.3 \%)$ & & & \\
\hline & & & & $\begin{array}{l}1 \\
2\end{array}$ & & \\
\hline
\end{tabular}

Figure 6 Subgroup analysis of association between level IV metastasis and DLN metastasis according to baseline characteristics. DLN, Delphian lymph node; ETE, extrathyroid extension; CLNM, central lymph node metastasis; OR, odds ratio; CI, confidence interval.

hold a relatively conservative view on prophylactic central compartment neck dissection (pCCND) (13-16). On the contrary, guidelines from China and Japan recommend the routine performance of pCCND. We found that only $14.7 \%$ of the cases were $\mathrm{cN} 1$ but CLNM occurred in $56.8 \%$ of patients, approving of the idea that neck ultrasonography has low diagnostic accuracy in identifying clinically relevant LNM (17). Therefore, to improve the accuracy of evaluation of the nodal status, more attention should be paid to the intraoperative information (18). Intraoperative frozen 
section examination can be conveniently used to guide a real-time diagnosis of the DLN status (11). According to DLN status, we found the rate of CLNM differs significantly in $\mathrm{cN} 0(87.7 \%$ vs. $43.2 \%)$ and $\mathrm{cN} 1(98.1 \%$ vs. $68.8 \%$ ). Moreover, the 2015 ATA consensus stated that it is appropriate not to perform a pCCND for patients with stage T1-T2, noninvasive, cN0 tumors (14), but our data of those patients showed that a relatively high rate of CLNM in DLN-positive group compared with DLN-negative group ( $86.4 \%$ vs. $42.7 \%)$. And on multivariate analysis, DLN metastasis was found to be an independent predictive factor for CLNM. Based on these results, the decision not to perform pCCND could leave behind metastatic lymph nodes, especially for patients who are confirmed to be DLN positive during operation.

Consistent with the study reported by Chai et al. (19), we found that patients with positive DLN involvement were more likely to have tumor located in upper portion/ isthmus $(36.5 \%$ vs. $25.1 \%, \mathrm{P}<0.001)$. This may reflect the DLN, which mainly collect lymphatic drainage of the upper portion and isthmus. Meanwhile, the upper thyroid lobe was recognized as metastasizing more easily to the lateral lymph node $(17,20)$. Since N1b represents a high risk of disease recurrence (21), $32 \%$ of patients had LLNM in our study. Other preliminary studies have also reported that DLN-positive patients were at higher risk of LLNM than DLN-negative patients $(7,10)$. This time our data provide strong evidence of the correlation between DLN metastasis and LLNM after fully adjusting the effect of potential confounding factors through multiple models. Furthermore, the subgroup analysis revealed that DLN metastasis was independently correlated with levels II, III, IV metastasis, particularly in lesions in the superior pole. Therefore, these findings suggest that the DLN status based on intraoperative frozen section examination is valuable to help guide tailoring the extent of dissection. When intraoperative frozen section examination suggests DLN is positive, we recommend performing a central compartment neck dissection. Lateral LN of ipsilateral levels II, III, IV should be meticulously evaluated. More precisely and comprehensively individual evaluation of lateral $\mathrm{LN}$ of each sub-level incorporating clinicopathologic features is still necessary in the future $(22,23)$.

To the best of our knowledge, our study covers the largest sample size on this issue with nearly 1,600 patients. Besides, rigorous analyses were executed to control important confounders. Nevertheless, this study still has several limitations. First, the nature of this retrospective study may contribute to a potential selection bias. It is hoped that a prospective randomized trial of lymph node dissection based on DLN status with long-term follow-ups may be helpful. Second, PTC types including rare variants were not included in the analysis.

\section{Conclusions}

In patients with PTC, DLN metastasis is an independent risk factor for CLNM and LLNM of levels II-IV. Our data strongly suggest that when DLN positivity was confirmed by intraoperative frozen section examination, meticulous intraoperative assessment for LNM is required to guide tailored treatment.

\section{Acknowledgments}

Funding: This work was supported by grants from Chongqing Science and Technology Committee (cstc2017shmsA1035).

\section{Footnote}

Reporting Checklist: The authors have completed the STROBE reporting checklist. Available at http://dx.doi. org/10.21037/gs-20-521

Data Sharing Statement: Available at http://dx.doi. org/10.21037/gs-20-521

Conflicts of Interest: All authors have completed the ICMJE uniform disclosure form (available at http://dx.doi. org/10.21037/gs-20-521). The authors have no conflicts of interest to declare.

Ethical Statement: the authors are accountable for all aspects of the work in ensuring that questions related to the accuracy or integrity of any part of the work are appropriately investigated and resolved. The study was conducted in accordance with the Declaration of Helsinki (as revised in 2013) and approved by ethical review board of The First Affiliated Hospital of Chongqing Medical University (reference number: 2020-182). Informed consent was obtained from all participants.

Open Access Statement: This is an Open Access article distributed in accordance with the Creative Commons Attribution-NonCommercial-NoDerivs 4.0 International 
License (CC BY-NC-ND 4.0), which permits the noncommercial replication and distribution of the article with the strict proviso that no changes or edits are made and the original work is properly cited (including links to both the formal publication through the relevant DOI and the license). See: https://creativecommons.org/licenses/by-nc-nd/4.0/.

\section{References}

1. Bray F, Ferlay J, Soerjomataram I, et al. Global cancer statistics 2018: GLOBOCAN estimates of incidence and mortality worldwide for 36 cancers in 185 countries. CA Cancer J Clin 2018;68:394-424.

2. Randolph GW, Duh QY, Heller KS, et al. The prognostic significance of nodal metastases from papillary thyroid carcinoma can be stratified based on the size and number of metastatic lymph nodes, as well as the presence of extranodal extension. Thyroid 2012;22:1144-52.

3. Podnos YD, Smith D, Wagman LD, et al. The implication of lymph node metastasis on survival in patients with well-differentiated thyroid cancer. Am Surg. 2005;71:731-4.

4. Wang TS, Sosa JA. Thyroid surgery for differentiated thyroid cancer - recent advances and future directions. Nat Rev Endocrinol 2018;14:670-83.

5. Miller JE, Al-Attar NC, Brown OH, et al. Location and Causation of Residual Lymph Node Metastasis After Surgical Treatment of Regionally Advanced Differentiated Thyroid Cancer. Thyroid 2018;28:593-600.

6. Hu JQ, Wen D, Ma B, et al. The extent of lymph node yield in central neck dissection can be affected by preoperative and intraoperative assessment and alter the prognosis of papillary thyroid carcinoma. Cancer Med 2020;9:1017-24.

7. Oh EM, Chung YS, Lee YD. Clinical significance of Delphian lymph node metastasis in papillary thyroid carcinoma. World J Surg 2013;37:2594-9.

8. Isaacs JD, Lundgren CI, Sidhu SB, et al. The Delphian lymph node in thyroid cancer. Ann Surg 2008;247:477-82.

9. Iyer NG, Kumar A, Nixon IJ, et al. Incidence and significance of Delphian node metastasis in papillary thyroid cancer. Ann Surg 2011;253:988-91.

10. Huang J, Sun W, Zhang H, et al. Use of Delphian lymph node metastasis to predict central and lateral involvement in papillary thyroid carcinoma: A systematic review and meta-analysis. Clin Endocrinol (Oxf) 2019;91:170-8.

11. Zhu J, Huang R, Hu D, et al. Individualized
Prediction Of Metastatic Involvement Of Lymph Nodes Posterior To The Right Recurrent Laryngeal Nerve In Papillary Thyroid Carcinoma. Onco Targets Ther 2019;12:9077-84.

12. Feng JW, Pan H, Wang L, et al. Determine the Optimal Extent of Thyroidectomy and Lymphadenectomy for Patients With Papillary Thyroid Microcarcinoma. Front Endocrinol (Lausanne) 2019;10:363.

13. Hughes DT, Rosen JE, Evans DB, et al. Prophylactic Central Compartment Neck Dissection in Papillary Thyroid Cancer and Effect on Locoregional Recurrence. Ann Surg Oncol 2018;25:2526-34.

14. Haugen BR, Alexander EK, Bible KC, et al. 2015 American Thyroid Association Management Guidelines for Adult Patients with Thyroid Nodules and Differentiated Thyroid Cancer: The American Thyroid Association Guidelines Task Force on Thyroid Nodules and Differentiated Thyroid Cancer. Thyroid 2016;26:1-133.

15. Gambardella C, Patrone R, Di Capua F, et al. The role of prophylactic central compartment lymph node dissection in elderly patients with differentiated thyroid cancer: a multicentric study. BMC Surg 2019;18:110.

16. Ywata de Carvalho A, Chulam TC, Kowalski LP. Longterm Results of Observation vs Prophylactic Selective Level VI Neck Dissection for Papillary Thyroid Carcinoma at a Cancer Center. JAMA Otolaryngol Head Neck Surg 2015;141:599-606.

17. Fraser S, Zaidi N, Norlen O, et al. Incidence and Risk Factors for Occult Level 3 Lymph Node Metastases in Papillary Thyroid Cancer. Ann Surg Oncol 2016;23:3587-92.

18. Raffaelli M, De Crea C, Sessa L, et al. Ipsilateral Central Neck Dissection Plus Frozen Section Examination Versus Prophylactic Bilateral Central Neck Dissection in cN0 Papillary Thyroid Carcinoma. Ann Surg Oncol 2015;22:2302-8.

19. Chai YJ, Kim SJ, Choi JY, et al. Papillary thyroid carcinoma located in the isthmus or upper third is associated with Delphian lymph node metastasis. World J Surg 2014;38:1306-11.

20. Zhang TT, Qi XZ, Chen JP, et al. The association between tumor's location and cervical lymph nodes metastasis in papillary thyroid cancer. Gland Surg 2019;8:557-68.

21. Sapuppo G, Palermo F, Russo M, et al. Latero-cervical lymph node metastases (N1b) represent an additional risk factor for papillary thyroid cancer outcome. J Endocrinol Invest 2017;40:1355-63.

22. Hei H, Song Y, Qin J. Individual prediction of lateral neck 
metastasis risk in patients with unifocal papillary thyroid carcinoma. Eur J Surg Oncol 2019;45:1039-45.

23. Dou Y, Chen Y, Hu D, et al. Development and validation

Cite this article as: Zhu J, Huang R, Yu P, Hu D, Ren H, Huang C, Su X. Clinical implications of Delphian lymph node metastasis in papillary thyroid carcinoma. Gland Surg 2021;10(1):73-82. doi: 10.21037/gs-20-521 of web-based nomograms for predicting lateral lymph node metastasis in patients with papillary thyroid carcinoma. Gland Surg 2020;9:172-82. 